\title{
Clinical Study \\ Peritoneal Dialysis in Dengue Shock Syndrome May Be Detrimental
}

\author{
Chulananda D. A. Goonasekera, Bhagya G. Thenuwara, and Ranjith P. V. Kumarasiri
}

Department of Anesthesiology, Faculty of Medicine, University of Peradeniya, Peradeniya 20400, Sri Lanka

Correspondence should be addressed to Chulananda D. A. Goonasekera, cgoonase@slt.lk

Received 7 October 2011; Revised 8 January 2012; Accepted 9 January 2012

Academic Editor: Marcel Tanner

Copyright () 2012 Chulananda D. A. Goonasekera et al. This is an open access article distributed under the Creative Commons Attribution License, which permits unrestricted use, distribution, and reproduction in any medium, provided the original work is properly cited.

Dengue shock syndrome is the most severe form of Dengue that can be fatal. Nonresponders to standard therapy need intensive care. This paper outlines the clinical features, complications, and outcomes of Dengue Shock Syndrome not responding to standard therapies and needing supportive care in a tertiary referral intensive care unit of a developing country. Nearly one-third die within 3 days of admission to ICU. Peritoneal dialysis predicts the worst outcomes.

\section{Introduction}

Dengue is an important arthropod-borne viral disease of humans [1]. Its presentation varies from a mild viral syndrome to hemorrhagic fever and severe shock. Its severe forms (hemorrhagic fever and shock syndrome) may lead to multiorgan involvement and death. Dengue Shock Syndrome (DSS) is characterized by a massive increase in systemic capillary permeability with consequent hypovolemia [2]. The mortality rate in Dengue Shock Syndrome ranges from 6 to 30 percent, most commonly reported in children. Fluid resuscitation is critical with added support for failing organs [3].

In the recent past, Sri Lanka has experienced a surge of the disease reaching epidemic proportions associated with a probable change in the virus strain to a more virulent form $[4,5]$. In this context we have also noted a rise in the number of cases with severe forms of the disease needing intensive care. The Peradeniya ICU is a tertiary referral centre and it attracts a large number of above patients with Dengue Shock Syndrome in the region who do not respond to standard therapy with intravenous fluids, antibiotics, and supportive care including inotropes.

\section{Methods}

We collected demographic data of all patients referred for intensive care between January 2009 and June 2010 (18 months) and analyzed their outcomes in relation to the complications of DSS that ensued during their ICU stay and the therapies given. The diagnosis of Dengue has been established upon clinical grounds (WHO guidelines 2009) and treated accordingly by the referring physicians before admission to the ICU. On admission to ICU, all have had fever, vomiting $78 \%$, abdominal pain $21 \%$, cough $5 \%$, and body ache among $3 \%$ of cases. Eleven patients have had serological tests and Dengue IgM was found to be positive amongst $72 \%$ and IgG in $50 \%$ of the tested. They all have had intravenous fluid therapies and antibiotics. During the course of therapy in ICU, $43.6 \%$ of cases received fresh frozen plasma, $21.8 \%$ cryoprecipitate, $32.7 \%$ blood, and $56.4 \%$ platelet transfusions. Furthermore, $36.4 \%$ of cases were mechanically ventilated for multiple reasons such as severe respiratory distress $\left(\mathrm{FiO}_{2}>60 \%\right), \mathrm{RR}>40 / \mathrm{min}$, myocardial failure needing inotropes for persistent hypotension despite adequate filling, that is, CVP above $12 \mathrm{~cm}$ of $\mathrm{H}_{2} \mathrm{O}$. The decision for peritoneal dialysis was based on low urine output $(<0.5 \mathrm{~mL} / \mathrm{kg} /$ hour$)$ detected over a period of time in an ICU as a trend despite resuscitation with fluids combined with a situation of fluid overload, high CVP, persistent hypotension, or severe ARDS. Neither the plasma creatinine (rather a delayed indicator of AKI) nor blood urea (not reliable in the presence of liver impairment) was used as a determining factor in implementing dialysis. Persistent hypotension in the presence of high CVP was interpreted as indication of myocardial involvement (with or without 


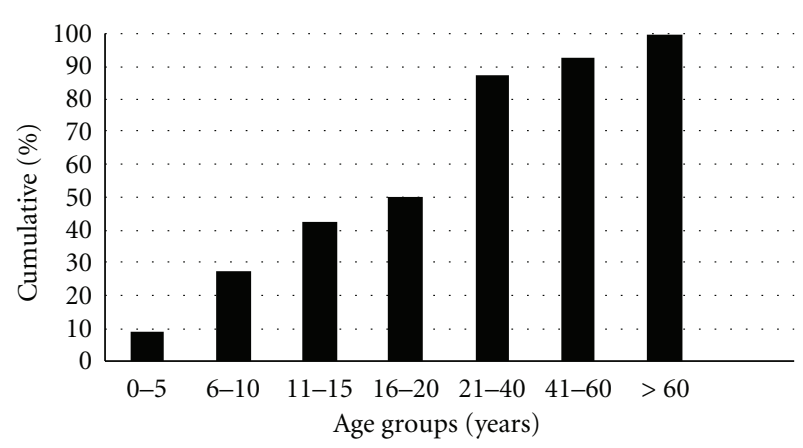

Figure 1: The cumulative age distribution of patients with Dengue shock syndrome needing intensive care.

TABLE 1: Was the death early or late in ICU?

\begin{tabular}{lcc}
\hline Day & No. of deaths & $\%$ \\
\hline 1 & 08 & $50 \%$ \\
2 & 03 & $19 \%$ \\
3 & 03 & $19 \%$ \\
5 & 01 & $06 \%$ \\
15 & 01 & $06 \%$ \\
\hline Total & 16 & $100 \%$ \\
\hline
\end{tabular}

relative bradycardia, ECG changes) and was supported with inotropes, usually a combination of dobutamine and noradrenaline and with mechanical ventilation if oxygenation was compromised.. Thus, our data is based on a group of severely ill patients diagnosed to be suffering from Dengue Shock Syndrome and continuing to deteriorate despite intravenous therapies administered in the wards. Liver failure regime, that is, oral/NG metronidazole and lactulose was introduced in patients who were found to have raised transaminases. It should be noted that the number of ICU beds available (10) in our hospital was approximately $1.5 \%$ of the total beds and this may have delayed the admission of some cases due to rationing.

\section{Results}

Between January 2009 and June 2010, 54 cases (25 male) diagnosed of Dengue Shock Syndrome were admitted for treatment to the Peradeniya ICU, a tertiary referral center. Half of them were aged 20 or below as shown in Figure 1. On admission, all had received prior intravenous fluid therapies. Their mean (SD) PCV was 44.5\% (5.5), WBC $7.7 \times 10^{9} / \mathrm{L}$ (4.6), Platelet count $2210^{9} / \mathrm{L}$ (17), respectively.

Of the 54, 16 died (mortality 29.6\%). Most deaths (88\%) occurred within 3 days of admission to the ICU (Table 1). The survivors needed intensive care for a median of 2 days (range 1-8) before being discharged to the referring wards for convalescence.

It is also noteworthy that $62.5 \%$ deaths occurred below the age 20 (see Table 2). Although we were unable to prove that risk of death was higher in children, a higher incidence
TABLE 2: Distribution of ICU deaths by age categories $(* 1$ missing data).

\begin{tabular}{lccc}
\hline & Live discharges & Deaths & Total \\
\hline$\leq 20$ years & 16 & 10 & 26 \\
$>20$ years & 21 & 06 & 27 \\
\hline Total & 37 & 16 & $53^{*}$ \\
\hline$\chi^{2}=1.66 ; \mathrm{df}=1 ; P=0.198$ (not significant). & &
\end{tabular}

of deaths (38\% as opposed to $22 \%$ ) was observed below the arbitrary cutoff age of 20 years.

To evaluate the risk of death according to the manifesting complications we performed a bivariate analysis and calculated the odds ratios (ORs) and confidence intervals (CI). OR and CI provide information on the strength (level of statistical significance) of association between the complications and the occurrence of deaths. Whereever the numbers of subjects were too small, the Fisher's exact test was used to calculate the $P$ value (Table 3 ).

We found that the complications of Dengue, namely, hemorrhage, pleural effusion, myocarditis, liver failure, and renal failure were independently linked with a 7-11 times higher risk of death compared to those without (Table 3). However, the wide confidence intervals indicated the higher variability of this observation.

The effect of treatment modality on the outcome (death) was evaluated with chi-square test (see Table 4). Chi-square test is widely used to evaluate the association between these predictor and outcome variables.

The results revealed that the treatment modalities, namely, the use of inotropes, mechanical ventilation, peritoneal dialysis, and the use of blood products were significantly associated with higher occurrence of deaths among these patients (Table 4). However, the use of steroids had no association with death.

Thereafter a discriminant analysis was used to classify the cases according to the values of categorical dichotomousdependent variables. This analysis assesses the relative importance of the independent variables in classifying the dependent variable.

The standardized canonical discriminant function coefficients identified mechanical ventilation and peritoneal dialysis as therapeutic modalities significantly associated with the deaths of dengue patients presenting with Dengue Shock Syndrome (Table 5).

A similar discriminant analysis was used to assess the relative importance of complications and outcome. Renal failure and hemorrhage were identified as complications significantly associated with deaths in Dengue Shock Syndrome (Table 6).

\section{Discussion}

We have evaluated the mortality risk factors amongst a cross-section of patients in Dengue Shock Syndrome not responding to standard therapies and as a consequence in a clinical scenario confounded by previous therapies before admission to ICU. Dengue Shock Syndrome is a dangerous 
TABLE 3: Complications ensued and outcomes amongst patients with Dengue shock syndrome in ICU (*Fisher's exact test).

\begin{tabular}{|c|c|c|c|c|c|c|c|}
\hline Complications & & Deaths & Discharges & Total & OR & $\mathrm{CI}$ & $P$ \\
\hline \multirow{3}{*}{ Hemorrhage } & Yes & 13 & 14 & 27 & \multirow{3}{*}{7.4} & \multirow{3}{*}{$1.8-30.7$} & \multirow{3}{*}{0.003} \\
\hline & No & 3 & 24 & 27 & & & \\
\hline & Total & 16 & 38 & 54 & & & \\
\hline \multirow{3}{*}{ Pleural effusion } & Yes & 13 & 14 & 27 & \multirow{3}{*}{7.4} & \multirow{3}{*}{$1.8-30.7$} & \multirow{3}{*}{0.003} \\
\hline & No & 3 & 24 & 27 & & & \\
\hline & Total & 16 & 38 & 54 & & & \\
\hline \multirow{3}{*}{ Myocarditis } & Yes & 8 & 6 & 14 & \multirow{3}{*}{5.3} & \multirow{3}{*}{$1.4-19.8$} & \multirow{3}{*}{$0.02^{*}$} \\
\hline & No & 8 & 32 & 40 & & & \\
\hline & Total & 16 & 38 & 54 & & & \\
\hline \multirow{3}{*}{ Renal failure } & Yes & 10 & 0 & 10 & & & \multirow{3}{*}{$<0.001^{*}$} \\
\hline & No & 06 & 38 & 44 & & & \\
\hline & Total & 16 & 38 & 54 & & & \\
\hline \multirow{3}{*}{ Liver failure } & Yes & 08 & 03 & 11 & \multirow{3}{*}{11.7} & \multirow{3}{*}{$2.2-54.1$} & \multirow{3}{*}{$0.001^{*}$} \\
\hline & No & 08 & 35 & 43 & & & \\
\hline & Total & 16 & 38 & 54 & & & \\
\hline
\end{tabular}

TABLE 4: Therapeutic modalities applied and outcomes of patients with Dengue Shock Syndrome receiving intensive care.

\begin{tabular}{|c|c|c|c|c|c|c|c|}
\hline Therapies & & Deaths & Discharges & Total & $\chi^{2}$ &. $\mathrm{df}$ & $P$ \\
\hline \multirow{3}{*}{ Inotropes } & Yes & 15 & 12 & 27 & \multirow{3}{*}{17.41} & \multirow{3}{*}{1} & \multirow{3}{*}{$<0.001$} \\
\hline & No & 01 & 26 & 27 & & & \\
\hline & Total & 16 & 38 & 54 & & & \\
\hline \multirow{3}{*}{ Mechanical ventilation } & Yes & 15 & 4 & 19 & \multirow{3}{*}{34.1} & \multirow{3}{*}{1} & \multirow{3}{*}{$<0.001$} \\
\hline & No & 1 & 34 & 35 & & & \\
\hline & Total & 16 & 38 & 54 & & & \\
\hline \multirow{3}{*}{ Liver failure regime } & Yes & 9 & 3 & 12 & \multirow{3}{*}{15.23} & \multirow{3}{*}{1} & \multirow{3}{*}{$<0.001$} \\
\hline & No & 7 & 35 & 42 & & & \\
\hline & Total & 16 & 38 & 54 & & & \\
\hline \multirow{3}{*}{ Peritoneal dialysis } & Yes & 8 & 0 & 8 & \multirow{3}{*}{22.3} & \multirow{3}{*}{1} & \multirow{3}{*}{$<0.001$} \\
\hline & No & 8 & 38 & 46 & & & \\
\hline & Total & 16 & 38 & 54 & & & \\
\hline \multirow{3}{*}{ Blood products } & 1 & 14 & 11 & 25 & \multirow{3}{*}{15.2} & \multirow{3}{*}{1} & \multirow{3}{*}{$<0.001$} \\
\hline & 2 & 2 & 27 & 29 & & & \\
\hline & Total & 16 & 38 & 54 & & & \\
\hline \multirow{3}{*}{ Steroids } & Yes & 7 & 13 & 20 & \multirow{3}{*}{0.353} & \multirow{3}{*}{1} & \multirow{3}{*}{0.553} \\
\hline & No & 9 & 24 & 33 & & & \\
\hline & Total & 16 & 37 & 53 & & & \\
\hline
\end{tabular}

complication of the dengue infection and is associated with high mortality. Almost one-third of our study group received blood transfusions to counter their bleeding manifestations. Thus, we were seeing the worst cases of the spectrum.

The pathogenesis of shock in dengue is complex. Increased vascular permeability, together with myocardial dysfunction and dehydration due to capillary leakage, contribute to the development of shock, with resultant multiorgan failure. The onset of shock can be dramatic, and its progression relentless. The diagnosis is largely clinical and is supported by serology and identification of viral material in the blood. No specific methods are available to predict outcome and progression. As observed by Singhi et al. [6] the choice of fluids, inotropes, and techniques of organ support and careful fluid management is the mainstay of management.

We have recorded a $30 \%$ mortality risk for this unique group of patients with Dengue Shock Syndrome who had received prior medical therapies and was admitted to Intensive Care with further deterioration. Unfortunately, the fact that $50 \%$ of the patients who succumbed did so within the first 24 hours of admission to the ICU indicates their moribund state upon referral to the ICU. It should be noted that the presenting clinical status of these patients to the ICU was confounded by a variety of treatment regimens that were applied before admission to the ICU. For example, in a typical patient admitted with respiratory distress and hypoxia, the clinical picture would easily be modified by 
Table 5: Standardized Canonical Discriminant functions between therapeutic modalities and outcome ( ${ }^{*}$ Wilk's lambda $=0.315$; $\mathrm{df}=$ 2; $P<0.001)$.

\begin{tabular}{lc}
\hline Variables & $\begin{array}{c}\text { Canonical discriminant function } \\
\text { coefficients }\end{array}$ \\
\hline Mechanical ventilation & $0.885^{*}$ \\
Peritoneal dialysis & $0.566^{*}$ \\
Liver failure regime & 0.474 \\
Blood products & 0.233 \\
Steroids & 0.215 \\
Inotropes & 0.192 \\
\hline
\end{tabular}

Table 6: Standardized Canonical Discriminant functions between complications and outcome.

\begin{tabular}{lc}
\hline Variables & $\begin{array}{c}\text { Canonical discriminant function } \\
\text { coefficients }\end{array}$ \\
\hline Renal failure & $.919^{*}$ \\
Liver failure & .476 \\
Pleural effusion & .417 \\
Hemorrhage & $.377^{*}$ \\
Myocarditis & .368 \\
\hline
\end{tabular}

${ }^{*}$ Wilk's lambda $=0.419 ; \mathrm{df}=02 ; P<0.001$.

overzealous hydration with colloids such as dextran or hetastarch prior to the ICU referral. In this study we have not been able to assess the influence of prior therapy on outcome due to poor medical records received at the admission to ICU. It is however the general impression of the authors, that the influence of prior therapies could be an important determinant of outcome, especially because some patients were noted to have had a cumulative dose of hetastarch exceeding $25 \mathrm{~mL} / \mathrm{kg}$ suggesting overload contributing to respiratory distress more than the disease itself. This is a very important aspect that cannot be overlooked in future studies of this nature.

There is a general impression that fatal dengue is commoner in the younger population compared to middle or old age $[7,8]$. Although we recorded $62.5 \%$ deaths amongst patients aged 20 or below, we have no statistical evidence to support the notion that mortality is higher amongst children. This is because our age distribution also indicated that $50 \%$ of the age cohort admitted for ICU care was above the age of 20. Peradeniya ICU is multidisciplinary and there are no age restrictions in its admission policy and hence we presume that our data represent the population with Dengue Shock Syndrome with no age bias.

In our study, from amongst the dead, $88 \%$ expired during first three days of ICU care and the highest death rate was reported on day 1 (50\% of total deaths). A similar study during an epidemic of dengue hemorrhagic fever in easternmost Indonesia showed a case fatality rate of $1.2 \%$ from a 172 suspected cases. They too observed that, the survivors needed a range of 1-8 days of ICU care [9] similar to the durations we observed. Another study from Mumbai during a dengue epidemic reported a case fatality rate of
$16.6 \%$ amongst pediatric patients suffering from Dengue Shock syndrome [10].

Bleeding has been identified as one of the dreaded manifestations of concern that complicates the outcome of dengue [11]. Although our canonical discriminant analysis indicated that hemorrhage and renal failure were the dreaded complications associated with death, from amongst its main therapeutic modalities only peritoneal dialysis (PD) was associated with death. This suggested that the use of blood products has effectively mitigated the effects of hemorrhage upon outcome. However, peritoneal dialysis did not show a similar effect suggesting that PD may not be the most appropriate modality of therapy in these moribund patients with multiorgan failure. It is also our clinical observation that PD cycles in Dengue patients produced a relatively large fluid retrieval without the use of additional measures such as dextrose in dialysate fluid. These large negative balances were corrected with the use of stored plasma intravenously. It is likely that these patients had ascites fluid that was also removed by each dialysis cycle and this may have simulated the main problem of dengue, the "capillary leak." Thus, peritoneal dialysis may have aggravated the clinical effects of continuing "capillary leak" leading to worsened outcomes. We had no facility for Continuous Renal Replacement Therapy (CRRT).

We also found that mechanical ventilation was also associated with death. Only $21 \%$ of patients who received mechanical ventilation in the ICU recovered in this study (4 out of 19). Since we had no facility for ECMO, it is difficult to comprehend whether mechanical ventilation is the best supportive mode of therapy to maintain oxygenation in DSS.

There was a significant relationship between dengue, complications, and the modes of therapies and outcome. Hemorrhage, pleural effusion, myocarditis, renal failure, and liver failure were all important predictors of the worst outcomes. A study conducted in Thailand implied the importance of detection of abnormal high transaminase enzyme among the patients with dengue infection since the consequently developed hepatic encephalopathy could be expected [12]. In our study 9 out of 12 patients who were treated with liver failure regime expired (75\%).

Dengue induced Acute Kidney Injury (AKI) comprising creatinine increase, proteinuria, glomerulonephritis, and haemolytic uremic syndrome has been reported $[13,14]$ and also dengue-haemorrhagic-fever-(DHF-) induced AKI even in the absence of shock, haemolysis, or rhabdomyolysis [15]. In our study 8 out of $8(100 \%)$ patients who were suspected as having renal failure expired despite peritoneal dialysis. Similar to our observation, Kuo et al. in the year 2002 reported a Dengue outbreak in Taiwan and noted that patients with renal failure (RF) carry a high mortality rate, that is, the morality rate RF group versus non-RF group was $28.6 \%$ against $1.2 \% ; P<0.001$ [16].

Acute reversible myocardial dysfunction is the commonest documented cardiac complication in dengue. The variable incidences of dengue myocarditis had been postulated to be due to variable immunopathogenesis secondary to variations in serotypes. Dengue myocarditis is generally reversible with favorable outcomes if diagnosed and treated 
early $[17,18]$. In our study 15 out of 27 patients $(55.55 \%)$ who were suspected of having dengue myocarditis were treated with inotropes but they died during their ICU stay.

It has been reported that corticosteroids were no more effective than the placebo or the no treatment protocol for reducing the number of deaths, the need for blood transfusion, or the number of serious complications [19] or in achieving a higher rise of the platelet count in dengue infection [20]. We have observed the same. No specific therapy has been shown to be effective in improving survival [21].

\section{Conclusion}

Our study indicates that during this dengue outbreak, patients in DSS who were not responding to standard therapies and admitted ICU had a 30\% risk of death. Peritoneal dialysis increases this risk to $100 \%$.

\section{Acknowledgments}

The authors are grateful to all ICU staff for providing information for this study. They thank Mr. Mahes Salgado for his contribution in the preparation of the paper.

\section{References}

[1] D. J. Gubler, "Dengue and dengue hemorrhagic fever," Clinical Microbiology Reviews, vol. 11, no. 3, pp. 480-496, 1998.

[2] T. N. Ngo, C. X. T. Phuong, R. Kneen et al., "Acute management of dengue shock syndrome: a randomized double-blind comparison of 4 intravenous fluid regimens in the first hour," Clinical Infectious Diseases, vol. 32, no. 2, pp. 204-213, 2001.

[3] S. Ranjit and N. Kissoon, "Dengue hemorrhagic fever and shock syndromes," Pediatric Critical Care Medicine, vol. 12, no. 1, pp. 90-100, 2010.

[4] G. N. Malavige, V. G. N. S. Velathanthiri, E. S. Wijewickrama et al., "Patterns of disease among adults hospitalized with dengue infections," QJM, vol. 99, no. 5, pp. 299-305, 2006.

[5] S. A. M. Kularatne, I. B. Gawarammana, and P. R. V. Kumarasiri, "Epidemiology, clinical features, laboratory investigations and early diagnosis of dengue fever in adults: a descriptive study in Sri Lanka," Southeast Asian Journal of Tropical Medicine and Public Health, vol. 36, no. 3, pp. 686692, 2005.

[6] S. Singhi, N. Kissoon, and A. Bansal, "Dengue and dengue hemorrhagic fever: management issues in an intensive care unit," Jornal de Pediatria, vol. 83, no. 2, supplement, pp. S22S35, 2007.

[7] T. K. Samsi, H. Wulur, D. Sugianto, C. R. Bartz, and R. Tan, "Some clinical and epidemiological observations on biologically confirmed dengue haemorrhagic fever," Paediatr Indones, vol. 30, no. 11-12, pp. 293-303, 1990.

[8] P. Gurugama, P. Garg, J. Perera, A. Wijewickrama, and S. Seneviratne, "Dengue viral infections," Indian Journal of Dermatology, vol. 55, no. 1, pp. 68-78, 2010.

[9] N. C. Sukri, K. Laras, T. Wandra et al., "Transmission of epidemic dengue hemorrhagic fever in easternmost Indonesia," American Journal of Tropical Medicine and Hygiene, vol. 68, no. 5, pp. 529-535, 2003.
[10] I. Shah, G. C. Deshpande, and P. N. Tardeja, "Outbreak of dengue in Mumbai and predictive markers for dengue shock syndrome," Journal of Tropical Pediatrics, vol. 50, no. 5, pp. 301-305, 2004.

[11] S. Shivbalan, K. Anandnathan, S. Balasubramanian, M. Datta, and E. Amalraj, "Predictors of spontaneous bleeding in dengue," Indian Journal of Pediatrics, vol. 71, no. 1, pp. 33-36, 2004.

[12] V. Wiwanitkit, "Liver dysfunction in Dengue infection: an analysis of the previously published Thai cases," JAMC, vol. 19, no. 1, pp. 10-12, 2007.

[13] P. Futrakul, V. Poshyachinda, and C. Mitrakul, "Renal involvement. And reticulo endothelial system clearance in dengue hemorrhagic fever," Journal of the Medical Association of Thailand, vol. 56, no. 1, pp. 33-39, 1973.

[14] V. Boonpucknavig, N. Bhamarapravati, and S. Boonpucknavig, "Glomerular changes in dengue hemorrhagic fever," Archives of Pathology and Laboratory Medicine, vol. 100, no. 4, pp. 206-212, 1976.

[15] E. Q. Lima, F. S. Gorayeb, J. R. Zanon, M. L. Nogueira, H. J. Ramalho, and E. A. Burdmann, "Dengue haemorrhagic feverinduced acute kidney injury without hypotension, haemolysis or rhabdomyolysis," Nephrology Dialysis Transplantation, vol. 22, no. 11, pp. 3322-3326, 2007.

[16] M. C. Kuo, P. L. Lu, J. M. Chang et al., "Impact of renal failure on the outcome of dengue viral infection," Clinical Journal of the American Society of Nephrology, vol. 3, no. 5, pp. 13501356, 2008.

[17] D. M. Salgado, J. M. Eltit, K. Mansfield et al., "Heart and skeletal muscle are targets of dengue virus infection," Pediatric Infectious Disease Journal, vol. 29, no. 3, pp. 238-242, 2010.

[18] V. Wiwanitkit, "Dengue myocarditis, rare but not fatal manifestation," International Journal of Cardiology, vol. 112, no. 1, article 122, 2006.

[19] R. Panpanich, P. Sornchai, and K. Kanjanaratanakorn, "Corticosteroids for treating dengue shock syndrome," Cochrane Database of Systematic Reviews, vol. 3, Article ID CD003488, 2006.

[20] S. A. M. Kularatne, C. Walathara, S. I. Mahindawansa et al., "Efficacy of low dose dexamethasone in severe thrombocytopenia caused by dengue fever: a placebo controlled study," Postgraduate Medical Journal, vol. 85, no. 1008, pp. 525-529, 2009.

[21] S. Rajapakse, "Dengue shock," Journal of Emergencies, Trauma and Shock, vol. 4, no. 1, pp. 120-127, 2011. 


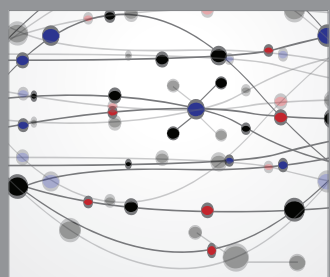

The Scientific World Journal
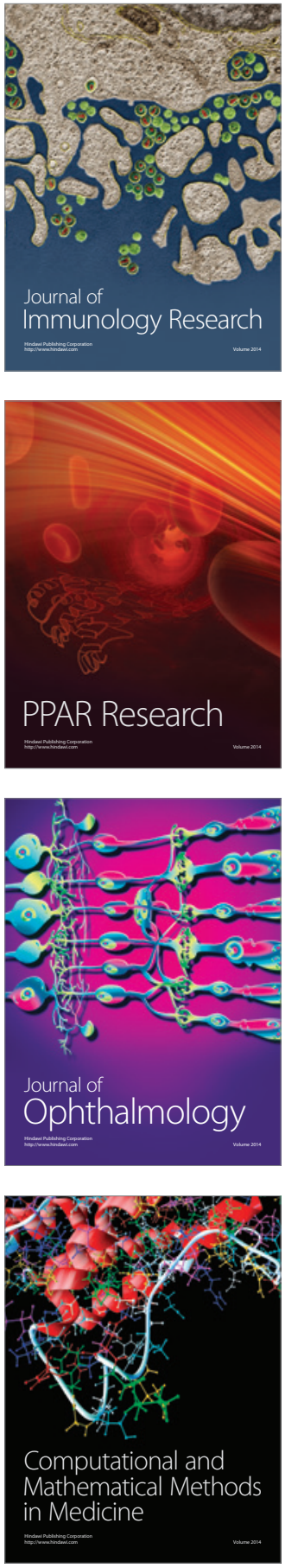

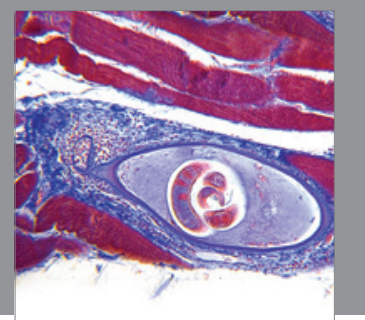

Gastroenterology

Research and Practice
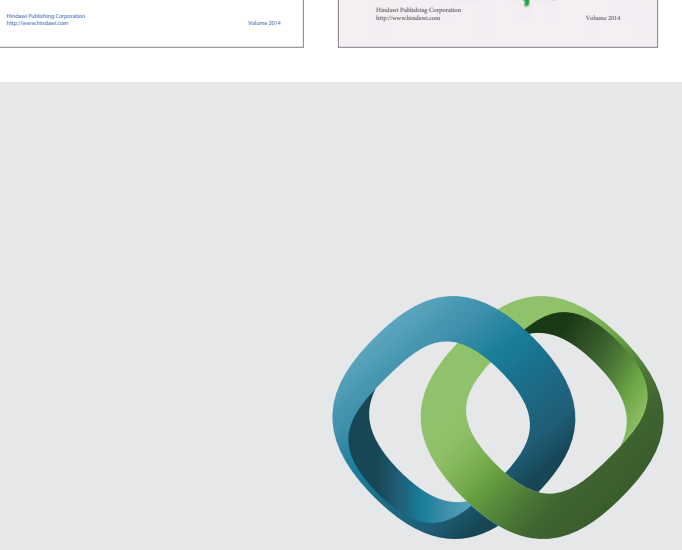

\section{Hindawi}

Submit your manuscripts at

http://www.hindawi.com
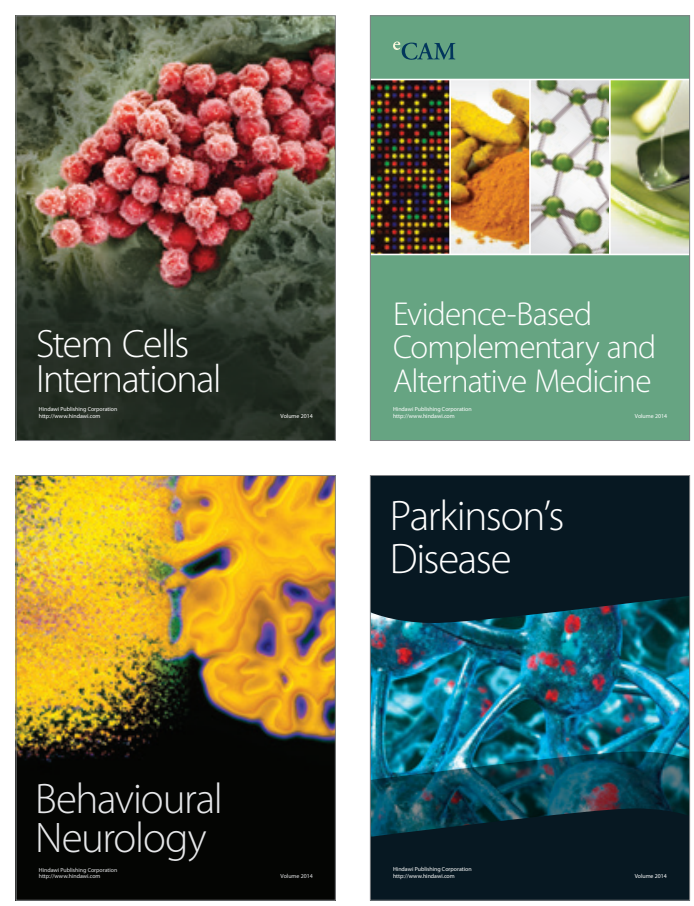

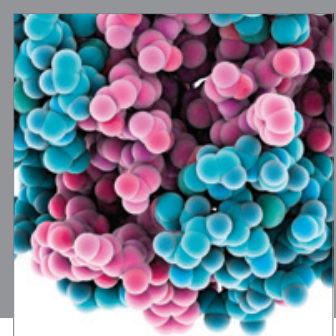

Journal of
Diabetes Research

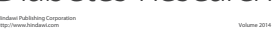

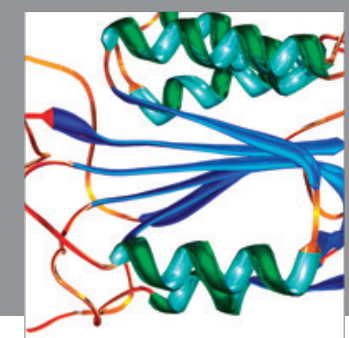

Disease Markers
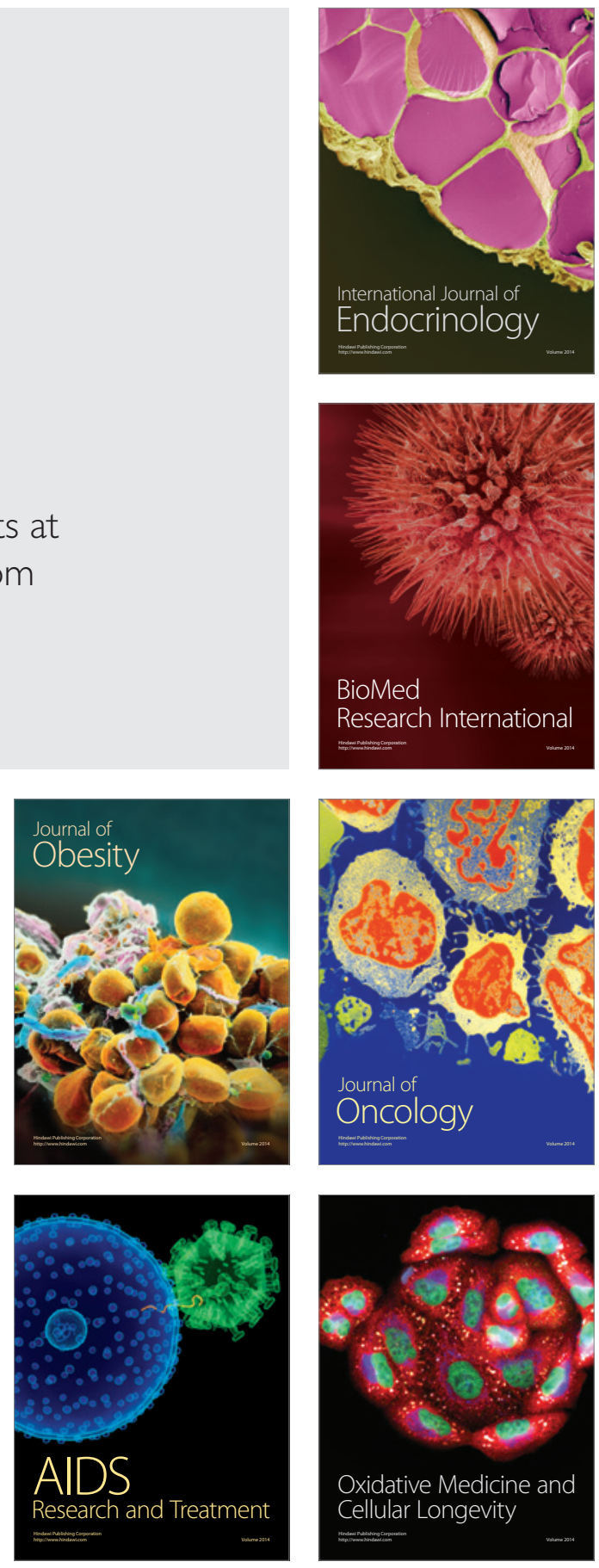